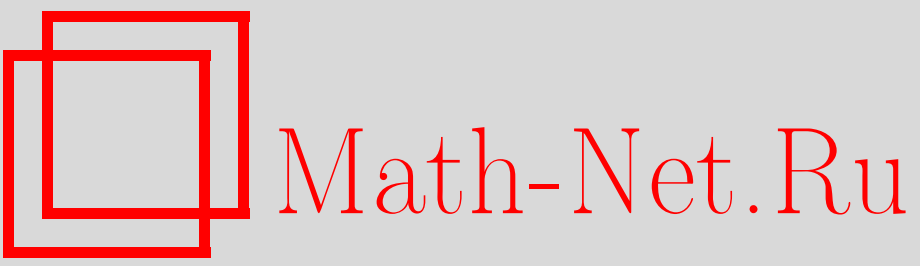

А. И. Алексеев, Аналитический инвариантный заряд в КХД с подавлением непертурбативных вкладов при больших $Q^{2}$, ТMФ, 2005, том 145, номер 2, 221239

DOI: https://doi.org/10.4213/tmf1898

Использование Общероссийского математического портала Math-Net.Ru подразумевает, что вы прочитали и согласны с пользовательским соглашением

http://www.mathnet.ru/rus/agreement

Параметры загрузки:

IP : 54.172 .240 .79

26 апреля 2023 г., 12:01:26 


\title{
АНАЛИТИЧЕСКИЙ ИНВАРИАНТНЫЙ ЗАРЯД В КХД С ПОДАВЛЕНИЕМ НЕПЕРТУРБАТИВНЫХ ВКЛАДОВ ПРИ БОЛЬШИХ $Q^{2}$
}

\begin{abstract}
На основе аналитического инвариантного заряда, полученного исходя из результатов стандартной теории возмушений в приближениях до четырехпетлевого, строится "синтетическая" модель инвариантного заряда в квантовой хромодинамике. В предложенной модели сохранен пертурбативный скачок на времениподобной полуоси комплексной плоскости $Q^{2}$, а непертурбативные вклады не только сокращают нефизические сингулярности теории возмущений в инфракрасной области, но и быстро убывают в ультрафиолетовой области. В рамках этой модели, с одной стороны, эффективная функция связи усилена в нуле (свойство дуальной сверхпроводимости вакуума квантовой хромодинамики), а с другой стороны, возникает динамическая масса глюона. В рамках рассматриваемого подхода задание параметра, соответствующего параметру натяжения струны, и нормировка, например, в точке $M_{\tau}$ полностью фиксируют синтетическую модель инвариантного заряда. При этом динамическая масса глюона $m_{\mathrm{g}}$ оказывается фиксированной и стабильной при увеличении числа петель исходного пертурбативного приближения.
\end{abstract}

Ключевые слова: аналитический подход, инфрракрасная область квантовой хромодинамики, бегущая константа связи, непертурбативные вклады.

\section{1. ВВЕДЕНИЕ}

Работа посвящена описанию модели инвариантного заряда [1] (или, иначе, бегушей константы связи) квантовой хромодинамики (КХД), в которой исходя из результатов пертурбативного исследования до четырехпетлевого уровня предпринята попытка учесть непертурбативные вклады таким образом, чтобы сократить нефизические пертурбативные сингулярности в инфракрасной (ИК) области и аккумулировать в его рамках основные представления о динамике КХД для всех масштабов.

Весьма привлекательным при этом представляется исходить из аналитического инвариантного заряда КХД и на этой основе строить новый инвариантный заряд, содержаший дополнительные непертурбативные члены. Мы будем строить разновидность моделей такого рода - "синтетическую" модель $\alpha_{\mathrm{syn}}\left(Q^{2}\right)$, которая, опираясь на результаты теории возмушений, используемой при описании области больших передач импульса $Q^{2}$

* Институт физики высоких энергий, Протвино, Московская обл., Россия. E-mail: alekseev@th1.ihep.su 
(физика малых расстояний), включает в себя непертурбативные члены, определяющие основные свойства КХД при малых $Q^{2}$ (физика больших расстояний) без резкой смены этих качественно различных режимов.

Аналитический подход в теории поля был сформулирован в конце 50-х г.г. ХХ в. в работах [2] на примерах квантовой электродинамики и других теорий. Для КХД аналитический подход применен в работах [3], [4]. Укажем на работы [5]-[7], в которых получили развитие аналитический подход и, в частности, аналитическая теория возмушений в КХД. Введение аналитической константы связи позволяет разрешить трудности, связанные с нефизическими сингулярностями теории возмушений в ИК-области. Эти сингулярности в аналитическом подходе сокращаются непертурбативными вкладами. В ультрафиолетовой (УФ) области непертурбативные вклады быстро убывают, а пертурбативные являются определяющими. Тем не менее большой интерес представляет поведение быстроубывающих в УФ-области непертурбативных вкладов, возникающих в результате процедуры "аналитизации". Их поведение оказывается важным и при построении нашей синтетической модели.

Если для однопетлевого случая разделение аналитической константы связи на пертурбативную и непертурбативную компоненты и поведение непертурбативной компоненты очевидны, то для многопетлевых случаев это не так. Для двухпетлевого случая такое разделение в явном виде и исследование поведения компонент аналитической константы связи проведены в работе [8], для трехпетлевого случая-в работах [9], [10], а для четырехпетлевого - в работах [11], [12]. Непертурбативные вклады были выделены в явном виде, и было получено их разложение по степеням $\Lambda^{2} / Q^{2}$, на основе которого развит эффективный метод прецизионного вычисления аналитической константы связи. Анализ импульсной зависимости аналитической константы связи [10], [12] при различных условиях сшивания на порогах тяжелых кварков показал, что значению константы взаимодействия $\alpha_{s}\left(M_{Z}^{2}\right)=0.118 \pm 0.002$ соответствует значение $\alpha_{\text {an }}\left(M_{\tau}^{2}\right)=0.294 \pm 0.011$, что заметно ниже имеюшихся оценок [13]-[15] значений величины $\alpha_{s}\left(M_{\tau}^{2}\right)$. Таким образом, соответствие среднемировому значению $[13] \alpha_{s}\left(M_{Z}^{2}\right)=0.1187_{-0.002}^{+0.002}$ побуждает

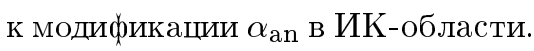

Как известно [3], аналитическая константа связи является конечной в нуле. В работах [11], [12] показано, что конечность аналитической константы связи в нуле является следствием свойства асимптотической свободы исходной теории возмущений, $\alpha_{\text {an }}^{\text {n-loop }}(0)=4 \pi / b_{0} \simeq 1.396$. Константа связи является конечной в нуле также при наличии "замораживания" взаимодействия [16], что является привлекательным с точки зрения феноменологии. Однако при таком ИК-поведении константы связи описание явлений конфайнмента и динамического нарушения киральной симметрии не является непосредственным. Поведение инвариантного заряда $\alpha_{\mathrm{V}} \sim 1 / Q^{2}$ при $Q^{2} \rightarrow 0$ в так называемой непертурбативной V-схеме “замороженной” КХД соответствует линейному запираюшему кварк-антикварковому статическому потенциалу с универсальным параметром натяжения струны $\sigma$. В КХД статический потенциал определяют калибровочно-инвариантным образом через вакуумное ожидание петли Вильсона. Рассматриваемая здесь синтетическая модель также относится к классу сингулярных, $\alpha_{\text {syn }} \sim 1 / Q^{2}$ при $Q^{2} \rightarrow 0$, однако имеет и свою дополнительную мотивацию, связанную с исследованием поведения непертурбативных вкладов при $Q^{2} \rightarrow \infty$. 
Основными методами непертурбативного исследования ИК-поведения функций Грина КХД и бегущей константы связи, которая может быть построена из этих функций, являются решение транкированных уравнений Дайсона-Швингера (ДШ) и вычисление функциональных интегралов на решетке. В работе [17] приводится сводка результатов таких непертурбативных исследований за последнее время; мы дополним ее работами, в которых использовались аналитические методы [18] и вычисления на решетке [19]. Разнообразие результатов для ИК-поведения $\alpha_{s}\left(Q^{2}\right)$ связано с типичной для калибровочных теорий вершинной зависимостью [17] определений бегушей константы связи при использовании зависящих от массы схем вычитания в импульсном пространстве, а также с различием методов транкирования системы уравнений ДШ. Кроме того, решение замкнутых уравнений (систем уравнений) требует, как правило, упрошаюших предположений, нередко нарушающих калибровочную симметрию, и аппроксимаций технического характера. Поэтому результаты исследования ИК-поведения $\alpha_{s}$ сильно отличаются друг от друга, и их не следует сравнивать непосредственно. Укажем на обзоры [20] по исследованию ИК-поведения функций Грина КХД, бегушей константы связи и их приложениям в физике адронов.

Возможность сингулярного поведения $\alpha_{s} \sim 1 / Q^{2}$ при $Q^{2} \rightarrow 0$, которую мы рассматриваем, исследовалась в ряде работ. В частности, в работах [21] ИК-поведение глюонных функций Грина исследовалось аналитическими методами в бездуховой аксиальной калибровке, в которой бегушая константа связи КХ Д определяется пропагатором глюона. В этих работах было показано, что сингулярное поведение глюонного пропагатора вида $D(Q) \sim 1 /\left(Q^{2}\right)^{2}$ при $Q^{2} \rightarrow 0$ возможно, но при этом необходимо отказаться от обычно используемого приближения трехглюонной вершинной функции ее продольной частью и учитывать вклад поперечной части трехглюонной вершинной функции определенного вида.

Структура настояшей работы такова. В разделе 2 рассматриваются основные идеи построения синтетической модели инвариантного заряда для всех $Q^{2}$ на однопетлевом уровне. В разделе 3 описывается построение аналитического инвариантного заряда для приближений стандартного вида исходной теории возмущений до четырехпетлевого и ее разделение на пертурбативную и непертурбативную компоненты. В разделе 4 на основе аналитического инвариантного заряда строится синтетическая минимальная модель инвариантного заряда с подавлением непертурбативных вкладов при больших $Q^{2}$. Производится фиксация параметров модели. В заключении обсуждаются результаты и формулируются основные выводы.

\section{2. СИНТЕТИЧЕСКАЯ МОДЕЛЬ ИНВАРИАНТНОГО ЗАРЯДА КХД НА ОДНОПЕТЛЕВОМ УРОВНЕ}

Рассмотрим следуюшую аддитивную модификацию однопетлевой бегущей константы связи КХД с помошью непертурбативных полюсных членов ${ }^{1)}$ :

$$
\alpha_{\mathrm{syn}}^{(1)}\left(Q^{2}\right)=\frac{4 \pi}{b_{0}}\left[\frac{1}{\ln \left(Q^{2} / \Lambda^{2}\right)}+\frac{\Lambda^{2}}{\Lambda^{2}-Q^{2}}+\frac{c \Lambda^{2}}{Q^{2}}+\frac{(1-c) \Lambda^{2}}{Q^{2}+m_{g}^{2}}\right],
$$

\footnotetext{
1) Однопетлевая модель рассматривалась в работах [22], [23] и окончательно сформулирована с фиксацией параметров из энергетических соображений в работах [24].
} 
где массовый параметр $m_{\mathrm{g}}$ определяется как

$$
m_{\mathrm{g}}^{2}=\frac{\Lambda^{2}}{c-1} .
$$

Здесь $Q^{2}$ - квадрат евклидова импульса, константа $b_{0}=11-2 n_{f} / 3\left(n_{f}\right.$ - число ароматов активных кварков), $\Lambda$ - размерный параметр однопетлевой модели КХД (степенны́е члены быстро убывают при больших $Q^{2}$ и на определении параметра $\Lambda$ как $\Lambda_{\mathrm{QCD}}$ практически не сказываются), $c$ - безразмерный параметр модели. Параметр $\Lambda$ может быть фиксирован, например, условием нормировки, а параметр модели $c$, как мы далее увидим, характеризует соотношение между параметром $\Lambda_{\mathrm{QCD}}$ и параметром натяжения струны $\sigma$ в струнных моделях ${ }^{2)}$. Для реалистического определения параметров $\Lambda_{\mathrm{QCD}}$, $\sigma$ и их связи необходимо, разумеется, выйти за рамки однопетлевого приближения.

Первый член уравнения (1) является решением уравнения ренормализационной группы (РГ) для бегушей константы связи $\mathrm{KX} Д \alpha_{s}\left(Q^{2}\right)$

$$
Q^{2} \frac{\partial \alpha_{s}\left(Q^{2}\right)}{\partial Q^{2}}=\beta\left(\alpha_{s}\right)
$$

в однопетлевом приближении, $\beta\left(\alpha_{s}\right) \simeq-\beta_{0} \alpha_{s}^{2}, \beta_{0}=b_{0} /(4 \pi)$. Вводя ренормализационно-инвариантный параметр $\Lambda$ (константу интегрирования дифференциального уравнения), получаем, что это решение,

$$
\alpha_{s}^{(1)}\left(Q^{2}\right)=\frac{4 \pi}{b_{0}} \frac{1}{\ln \left(Q^{2} / \Lambda^{2}\right)},
$$

имеет нефизическую сингулярность (полюс Ландау) при $Q^{2}=\Lambda^{2}$. Стремление к нулю выражения (4) при $Q^{2} \rightarrow \infty$ соответствует замечательному свойству асимптотической свободы [28] неабелевых калибровочных теорий, а увеличение $\alpha_{s}$ с уменьшением $Q^{2}$ (до некоторой критической величины или до бесконечности) может быть связано с решением проблемы конфайнмента.

Метод РГ позволяет просуммировать бесконечный класс диаграмм, восстанавливая при этом ренормализационную инвариантность. Однако в результате аналитическая структура в комплексной плоскости $Q^{2}$ существенно изменяется. Отдельные диаграммы являлись аналитическими функциями в комплексной плоскости $Q^{2}$ с разрезом вдоль действительной отрицательной полуоси, тогда как в выражении (4) помимо указанного разреза имеется полюс в точке $Q^{2}=\Lambda^{2}$. Второй член в формуле (1) является непертурбативным, $\Lambda^{2} \simeq \mu^{2} \exp \left\{-4 \pi /\left(b_{0} \alpha_{s}\left(\mu^{2}\right)\right)\right\} ;$ он введен для того, чтобы этот нефизический полюс сократить. Сумма двух первых членов в формуле (1) является аналитической функцией в комплексной плоскости $Q^{2}$ с разрезом от нуля до $-\infty$,

$$
\alpha_{\mathrm{an}}^{(1)}\left(Q^{2}\right)=\frac{4 \pi}{b_{0}}\left[\frac{1}{\ln \left(Q^{2} / \Lambda^{2}\right)}+\frac{\Lambda^{2}}{\Lambda^{2}-Q^{2}}\right] .
$$

\footnotetext{
${ }^{2)}$ В приближениях КХД, не учитывающих эффекты, связанные с массами тяжелых кварков, имеется только один размерный параметр. Например, это $\Lambda_{\mathrm{QCD}}$ для больших $Q^{2}$ или же параметр натяжения струны $\sigma$, адекватный при малых $Q^{2}$. В потенциальных моделях связь этих параметров исследуется при описании связанных состояний тяжелых кварков [25]-[27]. Рассматриваемая однопетлевая модель посредством параметра $c$ описывает связь параметра $\Lambda$ модели и параметра $\sigma$ модели.
} 
Эту функцию можно записать с помошью дисперсионного соотношения

$$
\alpha_{\mathrm{an}}^{(1)}\left(Q^{2}\right)=\int_{0}^{\infty} \frac{d \sigma \tilde{\rho}^{(1)}(\sigma)}{\sigma+Q^{2}}
$$

в котором функцию $\tilde{\rho}^{(1)}(\sigma)$ мы называем однопетлевой спектральной плотностью:

$$
\tilde{\rho}^{(1)}(\sigma)=\frac{4 \pi}{b_{0}} \frac{1}{\ln ^{2}\left(\sigma / \Lambda^{2}\right)+\pi^{2}} .
$$

Она может быть получена в результате аналитического продолжения $\alpha_{s}$ в пространство Минковского $Q^{2} \rightarrow-\sigma-i 0$ и вычисления ее мнимой части, $\tilde{\rho}^{(1)}(\sigma)=(1 / \pi) \operatorname{Im} \alpha_{s}^{(1)}(-\sigma-$ $i 0)$. При действительных $Q^{2}>0$ функция (5) является положительной убываюшей с ростом $Q^{2}$ функцией с максимальным значением в нуле $\alpha_{\mathrm{an}}^{(1)}(0)=4 \pi / b_{0}$. Второй, непертурбативный, член в формуле (5) не дает вклада в мнимую часть $\alpha_{\mathrm{an}}^{(1)}\left(Q^{2}\right)$ при переходе в пространство Минковского, так что $\tilde{\rho}^{(1)}(\sigma)=(1 / \pi) \operatorname{Im} \alpha_{\mathrm{an}}^{(1)}(-\sigma-i 0)$. Синтетическую модель (1) мы также можем записать в виде дисперсионного соотношения

$$
\alpha_{\mathrm{syn}}^{(1)}\left(Q^{2}\right)=\int_{-0}^{\infty} \frac{d \sigma \tilde{\rho}_{\mathrm{syn}}^{(1)}(\sigma)}{\sigma+Q^{2}} .
$$

Функцию $\tilde{\rho}_{\text {sуn }}^{(1)}(\sigma)$ мы назьваем однопетлевой спектральной плотностью для синтетической бегушей константы связи. Она содержит два дополнительных члена в виде $\delta$-функций:

$$
\tilde{\rho}_{\mathrm{syn}}^{(1)}(\sigma)=\tilde{\rho}^{(1)}(\sigma)+\frac{4 \pi}{b_{0}}\left[c \Lambda^{2} \delta(\sigma)+(1-c) \Lambda^{2} \delta\left(\sigma-m_{g}^{2}\right)\right] .
$$

Введение двух полюсных членов в точках $Q^{2}=0$ и $Q^{2}=-m_{\mathrm{g}}^{2}$ при значениях параметра $c>1$ не привело к возникновению особенностей функции $\alpha_{\mathrm{syn}}^{(1)}$ в комплексной плоскости $Q^{2}$ с разрезом вдоль отрицательной полуоси. В формуле (9) для спектральной плотности появились по сравнению с выражением (7) пертурбативного происхождения два дополнительных члена в виде $\delta$-функций, локализованных в точках $\sigma=0$ и $\sigma=m_{\mathrm{g}}^{2}$.

Приведем выражение (1) для $\alpha_{\mathrm{syn}}^{(1)}\left(Q^{2}\right)$ к явно ренормализационно-инвариантному виду. Это можно сделать, не решая дифференциальных РГ-уравнений. Записав для $\alpha_{\mathrm{syn}}^{(1)}\left(Q^{2}\right)$ условие нормировки, мы получаем уравнение для искомой зависимости параметра $\Lambda^{2}$ от значений $\alpha_{\mathrm{syn}}^{(1)}\left(\mu^{2}\right)$ и $\mu^{2}$ вида

$$
\alpha_{\mathrm{syn}}^{(1)}\left(\mu^{2}\right)=\frac{4 \pi}{b_{0}}\left[\frac{1}{\ln \left(\mu^{2} / \Lambda^{2}\right)}+\frac{\Lambda^{2}}{\Lambda^{2}-\mu^{2}}+\frac{c \Lambda^{2}}{\mu^{2}}+\frac{(1-c) \Lambda^{2}}{\mu^{2}+m_{g}^{2}}\right] .
$$

Из размерных соображений запишем

$$
\Lambda^{2}=\mu^{2} e^{-\varphi\left(a\left(\mu^{2}\right)\right)}
$$

причем $a\left(\mu^{2}\right)=\left(b_{0} / 4 \pi\right) \alpha_{\mathrm{syn}}^{(1)}\left(\mu^{2}\right)$. Тогда для функции $\varphi(a)$ получаем трансцендентное уравнение

$$
a=\frac{1}{\varphi(a)}+\frac{1}{1-e^{\varphi(a)}}+c e^{-\varphi(a)}-\frac{(c-1)^{2}}{1+(c-1) e^{\varphi(a)}}
$$


Решением этого уравнения является функция $\varphi(a)$, которая при $a \rightarrow+0$ имеет поведение $\varphi(a) \simeq 1 / a \rightarrow+\infty$ при всех значениях параметра $c$, соответствуюшее пертурбативной области. Поведение этого решения (при $c<1$ у функции $\varphi(a)$ имеется две ветви, из которых одна имеет правильное поведение в пертурбативной области) при $a \rightarrow$ $+\infty$ зависит от значений параметра $c$. При $c \geqslant 1 \varphi(a) \simeq-\ln (a / c) \rightarrow-\infty$. При $c<1$ функция $\varphi$ стремится к конечному значению, $\varphi(a) \rightarrow \varphi_{0}=-\ln (1-c)$. Бета-функцию $\beta_{\mathrm{syn}}\left(\alpha_{\text {syn }}\right)$ для синтетической бегушей константы связи мы найдем с помошью уравнения Гелл-Манна-Лоу

$$
Q^{2} \frac{\partial \alpha_{\mathrm{syn}}\left(Q^{2}\right)}{\partial Q^{2}}=\beta_{\mathrm{syn}}\left(\alpha_{\mathrm{syn}}\right) .
$$

Продифференцировав бегушую константу связи (1) с использованием уравнений (11), (12), получаем выражение

$$
\begin{aligned}
\beta_{\mathrm{syn}}\left(\alpha_{\mathrm{syn}}\right)= & \frac{4 \pi}{b_{0}}\left\{-a+\frac{1}{\varphi(a)}-\frac{1}{\varphi^{2}(a)}+\frac{1}{\left(1-e^{\varphi(a)}\right)^{2}}-\right. \\
& \left.-\frac{(c-1)^{2}}{\left(1+(c-1) e^{\varphi(a)}\right)^{2}}\right\}\left.\right|_{a=b_{0} \alpha_{\mathrm{syn}} /(4 \pi)} .
\end{aligned}
$$

Отсюда, зная поведение правильной ветви функции $\varphi(a)$ при $a \rightarrow 0, \infty$, можно найти асимптотики

$$
\beta_{\mathrm{syn}}\left(\alpha_{\mathrm{syn}}\right) \simeq-\frac{b_{0}}{4 \pi} \alpha_{\mathrm{syn}}^{2}+o\left(\alpha_{\mathrm{syn}}^{2}\right), \quad \alpha_{\mathrm{syn}} \rightarrow 0
$$

для всех значений $c$ и

$$
\begin{aligned}
& \beta_{\mathrm{syn}}\left(\alpha_{\mathrm{syn}}\right) \simeq-\alpha_{\mathrm{syn}}-\frac{4 \pi}{b_{0}} c(c-2)+o(1), \quad \alpha_{\mathrm{syn}} \rightarrow \infty \quad(c \geqslant 1), \\
& \beta_{\mathrm{syn}}\left(\alpha_{\mathrm{syn}}\right) \simeq-\frac{b_{0}}{4 \pi} \frac{\alpha_{\mathrm{syn}}^{2}}{(1-c)^{2}}+O\left(\alpha_{\mathrm{syn}}\right), \quad \alpha_{\mathrm{syn}} \rightarrow \infty \quad(c<1) .
\end{aligned}
$$

Сделав соответствуюшие разложения, можно убедиться в том, что при $\varphi \rightarrow 0$ особенность $\beta$-функции (14) является кажущейся. Можно убедиться также, что при всех $\alpha_{\text {syn }}>0$ функция $\beta_{\text {syn }}\left(\alpha_{\text {syn }}\right)$ является отрицательно определенной (для правильной ветви при $0<c<1)$.

Три последних члена синтетической бегушей константы (1) с учетом (2) запишем в виде

$$
\begin{aligned}
\alpha_{\mathrm{syn}}^{\mathrm{npt}(1)}\left(Q^{2}\right) & =\frac{4 \pi}{b_{0}}\left[\frac{\Lambda^{2}}{\Lambda^{2}-Q^{2}}+\frac{c \Lambda^{2}}{Q^{2}}+\frac{(1-c) \Lambda^{2}}{Q^{2}+m_{g}^{2}}\right]= \\
& =\frac{4 \pi}{b_{0}} \frac{c \Lambda^{6}}{Q^{2}\left(\Lambda^{2}-Q^{2}\right)\left(\Lambda^{2}+(c-1) Q^{2}\right)} .
\end{aligned}
$$

При $a \rightarrow+0$ согласно уравнению (12) функция $\phi(a) \simeq 1 / a$, так что для $\Lambda$ из (11) получаем $\Lambda^{2} \simeq \mu^{2} e^{-1 / a}$ при $\alpha_{\text {syn }}\left(\mu^{2}\right)=\left(4 \pi / b_{0}\right) a \rightarrow+0$, и поэтому члены уравнения $(17)$ следует рассматривать как непертурбативные. Из формулы (17) видим, что при $c=0$ синтетическая бегушая константа связи (1) в однопетлевом приближении сводится к пертурбативному выражению (4). Сингулярный в нуле (ответственный за ИК-усиление) 
член обрашается в нуль, а массовый член сокрашается с членом, возникшим в результате процедуры аналитизации.

При $c=1$ в синтетической модели пертурбативный полюс Ландау сокращается, массовьци член исчезает, сингулярный член присутствует. При больших $Q^{2}$ непертурбативные члены вида $\sim 1 / Q^{2}$ сокрашаются, поведение непертурбативных вкладов таково:

$$
\alpha_{\mathrm{syn}}^{\mathrm{npt}(1)}\left(Q^{2}\right)=\frac{4 \pi}{b_{0}}\left[-\frac{\Lambda^{4}}{Q^{4}}\right]+O\left(\frac{\Lambda^{6}}{Q^{6}}\right) .
$$

При невыделенных значениях параметра $c(c \neq 0,1)$ непертурбативный "хвост" при больших $Q^{2}$ имеет поведение

$$
\alpha_{\mathrm{syn}}^{\mathrm{npt}(1)}\left(Q^{2}\right)=\frac{4 \pi}{b_{0}}\left[\frac{c}{1-c} \frac{\Lambda^{6}}{Q^{6}}\right]+O\left(\frac{\Lambda^{8}}{Q^{8}}\right) .
$$

Как видно из формулы (19), непертурбативные вклады убывают при больших $Q^{2}$ весьма быстро.

Таким образом, синтетическая бегушая константа связи КХД на однопетлевом уровне имеет следующие интересные свойства.

- По построению как функция $Q^{2}$ она имеет при $c \geqslant 1$ аналитическую структуру, соответствуюшую условию причинности, т.е. является голоморфной функцией в комплексной плоскости $Q^{2}$ с разрезом вдоль действительной оси от нуля до $-\infty$.

- Как функция значения в точке нормировки $\alpha_{\mathrm{Syn}}\left(\mu^{2}\right)$ она имеет сушественную особенность в нуле, асимптотическое разложение ее непертурбативной части по $\alpha_{\mathrm{syn}}\left(\mu^{2}\right)$ при $\alpha_{\mathrm{syn}}\left(\mu^{2}\right) \rightarrow+0$ равно нулю, что дает соответствие исходной теории возмущений.

- В УФ-области она соответствует обычному результату теории возмущений (с учетом ренормализационной инвариантности) с точностью до быстроубываюших степенных членов. При $c=1$ непертурбативные члены имеют поведение вида $\sim 1 /\left(Q^{2}\right)^{2}$ при $Q^{2} \rightarrow \infty$, а при $c \neq 1$ - поведение вида $\sim 1 /\left(Q^{2}\right)^{3}$ при $Q^{2} \rightarrow \infty$.

- В ИК-области при $c \neq 0$ она не имеет нефизических сингулярностей теории возмушений. В зависимости от значений параметра $c$ модель отвечает четырем качественно различным случаям. При $c>1$ имеются массовый член и член, отвечающий ИК-усилению. При $c=1$ имеется фиксированньй член с ИК-усилением, а массовый член отсутствует. При $0<c<1$ имеем член с ИК-усилением и тахионный член, а при $c<0$ ситуация совсем экзотическая: имеем "антиконфайнмент" и тахионный член.

Далее мы увидим, в какой степени эти свойства модифицируются при переходе к многопетлевым случаям. Важно отметить, что в однопетлевом случае мы имеем не только представление (8), но, во-первых, непертурбативные вклады выделены из синтетической бегущей константы связи в явном виде:

$$
\alpha_{\text {syn }}^{(1)}\left(Q^{2}\right)=\alpha^{\mathrm{pt}(1)}\left(Q^{2}\right)+\alpha_{\mathrm{syn}}^{\mathrm{npt}(1)}\left(Q^{2}\right) ;
$$

во-вторых, для непертурбативных вкладов имеется простая формула. При больших $Q^{2}$ $\left(Q^{2}>\Lambda^{2}\right)$ эти вклады могут быть представлены в виде ряда

$$
\alpha_{\mathrm{syn}}^{\mathrm{npt}(1)}\left(Q^{2}\right)=-\frac{4 \pi}{b_{0}}\left(\frac{\Lambda^{2}}{Q^{2}}\right)^{3} \sum_{n=0}^{\infty}\left(1-(1-c)^{-n-1}\right)\left(\frac{\Lambda^{2}}{Q^{2}}\right)^{n} .
$$


При малых $Q^{2}\left(Q^{2}<\Lambda^{2}\right)$ имеем разложение

$$
\alpha_{\mathrm{syn}}^{\mathrm{npt}(1)}\left(Q^{2}\right)=\frac{4 \pi}{b_{0}} \frac{\Lambda^{2}}{Q^{2}} \sum_{n=0}^{\infty}\left(1-(1-c)^{n+1}\right)\left(\frac{Q^{2}}{\Lambda^{2}}\right)^{n} .
$$

Выделенным из соображений симметрии случаем является случай $c=2$,

$$
\begin{aligned}
\alpha_{\mathrm{syn}}^{\mathrm{npt}(1)}\left(Q^{2}\right) & =\frac{4 \pi}{b_{0}}\left[\frac{\Lambda^{2}}{\Lambda^{2}-Q^{2}}+\frac{2 \Lambda^{2}}{Q^{2}}-\frac{\Lambda^{2}}{\Lambda^{2}+Q^{2}}\right]= \\
& =\frac{4 \pi}{b_{0}} \frac{2 \Lambda^{2}}{Q^{2}} \frac{\Lambda^{4}}{\Lambda^{4}-Q^{4}},
\end{aligned}
$$

для которого непертурбативные вклады являются нечетной функцией $Q^{2}$ :

$$
\alpha_{\mathrm{syn}}^{\mathrm{npt}(1)}\left(-Q^{2}\right)=-\alpha_{\mathrm{syn}}^{\mathrm{npt}(1)}\left(Q^{2}\right)
$$

В этом случае как в УФ-, так и в ИК-разложениях отсутствуют члены четных степеней $Q^{2}$. В частности, в ИК-разложении отсутствует кулоновская мода. Укажем для полноты на случай $c=\infty$, для которого

$$
\alpha_{\mathrm{syn}}^{\mathrm{npt}(1)}\left(Q^{2}\right)=\frac{4 \pi}{b_{0}} \frac{\Lambda^{6}}{Q^{4}\left(\Lambda^{2}-Q^{2}\right)}
$$

и сингулярность в ИК-области более сильная: $\alpha_{\mathrm{syn}}^{(1)}\left(Q^{2}\right) \sim 1 /\left(Q^{2}\right)^{2}, Q^{2} \rightarrow 0$.

\section{3. МНОГОПЕТЛЕВОЙ АНАЛИТИЧЕСКИЙ ИНВАРИАНТНЫЙ ЗАРЯД КХД}

В многопетлевом случае РГ-уравнение (3) для бегушей константы связи $\alpha_{s}\left(Q^{2}\right)$ имеет вид

$$
Q^{2} \frac{\partial \alpha_{s}\left(Q^{2}\right)}{\partial Q^{2}}=\beta\left(\alpha_{s}\right)=-\beta_{0} \alpha_{s}^{2}-\beta_{1} \alpha_{s}^{3}-\beta_{2} \alpha_{s}^{4}-\beta_{3} \alpha_{s}^{5}+O\left(\alpha_{s}^{6}\right),
$$

где коэффициенты (см. [28], [29])

$$
\begin{aligned}
\beta_{0}= & \frac{1}{4 \pi} b_{0}, \quad b_{0}=11-\frac{2}{3} n_{f}, \\
\beta_{1}= & \frac{1}{8 \pi^{2}} b_{1}, \quad b_{1}=51-\frac{19}{3} n_{f}, \\
\beta_{2}= & \frac{1}{128 \pi^{3}} b_{2}, \quad b_{2}=2857-\frac{5033}{9} n_{f}+\frac{325}{27} n_{f}^{2} \\
\beta_{3}= & \frac{1}{256 \pi^{4}} b_{3}, \quad b_{3}=\frac{149753}{6}+3564 \zeta_{3}- \\
& -\left(\frac{1078361}{162}+\frac{6508}{27} \zeta_{3}\right) n_{f}+\left(\frac{50065}{162}+\frac{6472}{81} \zeta_{3}\right) n_{f}^{2}+\frac{1093}{729} n_{f}^{3} .
\end{aligned}
$$

Здесь $n_{f}$ - число ароматов активных кварков, $\zeta$ - дзета-функция Римана, $\zeta_{3}=\zeta(3)=$ $1.202056903 \ldots$... Коэффициенты $\beta_{0}, \beta_{1}$ не зависят от выбора схемы перенормировки; следуюшие коэффициенты зависят. Приведенные выражения соответствуют выбору схемы $\overline{M S}$, ими мы и будем пользоваться при численных расчетах. 
Решение уравнения (25) для $\alpha_{s}\left(Q^{2}\right)$ при $L=\ln \left(Q^{2} / \Lambda^{2}\right) \rightarrow \infty$ записывают [30] в виде

$$
\begin{aligned}
\alpha_{s}\left(Q^{2}\right)= & \frac{1}{\beta_{0} L}\left\{1-\frac{\beta_{1}}{\beta_{0}^{2} L} \ln L+\frac{\beta_{1}^{2}}{\beta_{0}^{4} L^{2}}\left[\ln ^{2} L-\ln L-1+\frac{\beta_{0} \beta_{2}}{\beta_{1}^{2}}\right]-\right. \\
& -\frac{\beta_{1}^{3}}{\beta_{0}^{6} L^{3}}\left[\ln ^{3} L-\frac{5}{2} \ln ^{2} L-\left(2-\frac{3 \beta_{0} \beta_{2}}{\beta_{1}^{2}}\right) \ln L+\right. \\
& \left.\left.+\frac{1}{2}-\frac{\beta_{0}^{2} \beta_{3}}{2 \beta_{1}^{3}}\right]+O\left(\frac{1}{L^{4}}\right)\right\} .
\end{aligned}
$$

Отрезки ряда (27) с членами вида до $1 / L^{n}$ включительно $(n=1,2,3,4)$ в наших дальнейших построениях мы будем называть пертурбативной компонентой инвариантного заряда в $n$-петлевом приближении и обозначать как $\alpha^{\mathrm{pt}}\left(Q^{2}\right)$. Она может быть записана в виде

$$
\begin{gathered}
\alpha^{\mathrm{pt}}\left(Q^{2}\right)=\frac{4 \pi}{b_{0}} a(x), \\
a(x)=\frac{1}{\ln x}-b \frac{\ln (\ln x)}{\ln ^{2} x}+b^{2}\left[\frac{\ln ^{2}(\ln x)}{\ln ^{3} x}-\frac{\ln (\ln x)}{\ln ^{3} x}+\frac{\kappa}{\ln ^{3} x}\right]- \\
-b^{3}\left[\frac{\ln ^{3}(\ln x)}{\ln ^{4} x}-\frac{5}{2} \frac{\ln ^{2}(\ln x)}{\ln ^{4} x}+(3 \kappa+1) \frac{\ln (\ln x)}{\ln ^{4} x}+\frac{\bar{\kappa}}{\ln ^{4} x}\right] .
\end{gathered}
$$

Здесь $x=Q^{2} / \Lambda^{2}$, а коэффициенты

$$
\begin{aligned}
& b=\frac{\beta_{1}}{\beta_{0}^{2}}=\frac{2 b_{1}}{b_{0}^{2}}, \\
& \kappa=-1+\frac{\beta_{0} \beta_{2}}{\beta_{1}^{2}}=-1+\frac{b_{0} b_{2}}{8 b_{1}^{2}}, \\
& \bar{\kappa}=\frac{1}{2}-\frac{\beta_{0}^{2} \beta_{3}}{2 \beta_{1}^{3}}=\frac{1}{2}-\frac{b_{0}^{2} b_{3}}{16 b_{1}^{3}} .
\end{aligned}
$$

Величины $b_{0}, b, \kappa, \bar{\kappa}$ зависят от $n_{f}$. В стандартной картине сшивания решений на порогах тяжелых кварков параметр $\Lambda$ также становится зависяшим от $n_{f}{ }^{3)}$.

Аналитическая константа связи определяется посредством интегрального представления

$$
a_{\mathrm{an}}(x)=\frac{1}{\pi} \int_{0}^{\infty} \frac{d \sigma}{x+\sigma} \rho(\sigma),
$$

в котором спектральная плотность $\rho(\sigma)=\operatorname{Im} a_{\text {an }}(-\sigma-i 0)$. В аналитическом подходе полагают ${ }^{4)} \operatorname{Im} a_{\text {an }}(-\sigma-i 0)=\operatorname{Im} a(-\sigma-i 0)$. В результате от функции $a(x)$ вида $(29)$ с нефизическими сингулярностями на положительной действительной полуоси комплексной плоскости $Q^{2}$ приходим к $a_{\text {an }}(x)$ вида $(31)$, являющейся однозначной аналитической

\footnotetext{
${ }^{3)}$ В рамках теории возмущений параметр $\Lambda$ зависит также от выбора схемы перенормировки. Для исследования отклонений непертурбативных моделей и их следствий от пертурбативных величин предпочтительным является выбор стандартной схемы перенормировки $\overline{M S}$.

4) Мы рассматриваем именно такой вариант аналитизации. Константу связи, полученную в результате описанной процедуры, мы и называем аналитической константой связи.
} 
функцией в комплексной плоскости $Q^{2}$ с разрезом от нуля до - $\infty$ (при стандартном определении разрезов логарифмической функции). В работах [11], [12] до четырехпетлевого уровня получено разделение аналитической константы связи на пертурбативную и непертурбативную компоненты,

$$
\alpha_{\mathrm{an}}\left(Q^{2}\right)=\alpha^{\mathrm{pt}}\left(Q^{2}\right)+\alpha_{\mathrm{an}}^{\mathrm{npt}}\left(Q^{2}\right) .
$$

В формуле (32) под пертурбативной компонентой $\alpha^{\mathrm{pt}}\left(Q^{2}\right)$ подразумевается исходное (в данном случае стандартное) решение уравнения РГ до четырехпетлевого, а $\alpha_{\mathrm{an}}^{\mathrm{npt}}\left(Q^{2}\right)-$ это то, что возникло дополнительно в результате процедуры аналитизации. В указанных работах для непертурбативной компоненты получено явное выражение, полезное, в частности, для аналитического продолжения функции $\alpha_{\text {an }}$ в комплексную плоскость $Q^{2}$, а также разложение в степенной ряд

$$
\alpha_{\mathrm{an}}^{\mathrm{npt}}\left(Q^{2}\right)=\frac{4 \pi}{b_{0}} \sum_{n=1}^{\infty} c_{n}\left(\frac{\Lambda^{2}}{Q^{2}}\right)^{n},
$$

где

$$
\begin{aligned}
c_{n}= & -1+b n[1-\gamma-\ln (n)]-\frac{1}{2} b^{2} n^{2}\left[1-\frac{\pi^{2}}{6}+\kappa+(1-\gamma-\ln (n))^{2}\right]+ \\
& +\frac{1}{6} b^{3} n^{3}\left[2+\frac{5}{2} \kappa+\bar{\kappa}-2 \zeta_{3}+(1-\gamma-\ln (n))^{3}+\right. \\
& \left.+3(1-\gamma-\ln (n))\left(1-\frac{\pi^{2}}{6}+\kappa\right)\right] .
\end{aligned}
$$

Здесь $\gamma \simeq 0.5772$ - постоянная Эйлера. Ряд (33) равномерно сходится при $Q^{2}>\Lambda^{2}$, он является основой эффективного метода прецизионного исследования $\alpha_{\mathrm{an}}\left(Q^{2}\right)$ при всех $Q>\Lambda$. В табл. 1 приведены значения коэффициентов $c_{n}$ для первых пяти членов ряда (33) в интересуюшем нас случае $n_{f}=3$. Отметим такие важные свойства разложения (33), как высокую петлевую стабильность коэффициента лидируюшего члена и медленный рост коэффициентов $c_{n}$ с увеличением порядкового номера $n$.

ТАБлицА 1. Зависимость коэффициентов $c_{n}$ и петлевых вкладов в эти коэффициенты от порядкового номера для 1-4-петлевых случаев, $n_{f}=3$.

\begin{tabular}{|c|c|c|c|c|c|c|c|}
\hline$n$ & $c_{n}^{1 \text {-loop }}$ & $\Delta_{n}^{2 \text {-loop }}$ & $\Delta_{n}^{3 \text {-loop }}$ & $\Delta_{n}^{4-\text { loop }}$ & $c_{n}^{2 \text {-loop }}$ & $c_{n}^{3 \text {-loop }}$ & $c_{n}^{4 \text {-loop }}$ \\
\hline 1 & -1.0 & 0.33405 & 0.01608 & -0.07825 & -0.66595 & -0.64987 & -0.72812 \\
2 & -1.0 & -0.42724 & 0.19624 & -0.37379 & -1.42724 & -1.23101 & -1.60480 \\
3 & -1.0 & -1.60196 & -0.63626 & -1.28115 & -2.60196 & -3.23823 & -4.51937 \\
4 & -1.0 & -3.04517 & -3.48651 & -5.07338 & -4.04517 & -7.53168 & -12.60506 \\
5 & -1.0 & -4.68801 & -9.19185 & -16.30462 & -5.68801 & -14.87987 & -31.18449 \\
\hline
\end{tabular}




\section{4. МНОГОПЕТЛЕВОЙ СИНТЕТИЧЕСКИЙ ИНВАРИАНТНЫЙ ЗАРЯД КХД}

Обобщим однопетлевую синтетическую модель на многопетлевые случаи. Итак, модифицируем аналитическую константу связи путем введения двух дополнительных непертурбативных членов: сингулярного в нуле члена вида $\sim 1 / Q^{2}$ и массового члена вида $\sim 1 /\left(Q^{2}+m_{\mathrm{g}}^{2}\right)$. В результате мы приходим к выражению

$$
\alpha_{\mathrm{syn}}\left(Q^{2}\right)=\alpha_{\mathrm{an}}\left(Q^{2}\right)+\frac{4 \pi}{b_{0}}\left[\frac{c \Lambda^{2}}{Q^{2}}-\frac{d \Lambda^{2}}{Q^{2}+m_{\mathrm{g}}^{2}}\right] ;
$$

в него помимо $\Lambda$ вошли три дополнительных параметра: $c, d$ и $m_{\mathrm{g}}\left(m_{\mathrm{g}} \equiv m_{\Lambda} \Lambda\right)$, которые мы считаем ненулевыми. Задачу определения этих параметров решаем следующим образом. При больших $Q^{2}\left(Q^{2}>\Lambda^{2}\right)$ мы можем с помощью разложения (33) записать

$$
\begin{aligned}
\alpha_{\text {Syn }}\left(Q^{2}\right)= & \alpha^{\mathrm{pt}}\left(Q^{2}\right)+\frac{4 \pi}{b_{0}}\left[\left(c_{1}+c-d\right) \frac{\Lambda^{2}}{Q^{2}}+\left(c_{2}+d m_{\Lambda}^{2}\right)\left(\frac{\Lambda^{2}}{Q^{2}}\right)^{2}+\right. \\
& \left.+\left(c_{3}-d m_{\Lambda}^{4}\right)\left(\frac{\Lambda^{2}}{Q^{2}}\right)^{3}\right]+O\left(\left(\frac{\Lambda^{2}}{Q^{2}}\right)^{4}\right) .
\end{aligned}
$$

Потребуем, чтобы непертурбативные вклады при больших $Q^{2}$ были минимальными, т.е. чтобы члены вида $\sim 1 / Q^{2}, \sim 1 /\left(Q^{2}\right)^{2}$ в выражении (36) отсутствовали ${ }^{5)}$. Тогда два параметра из трех оказываются фиксированными посредством уравнений

$$
d=c+c_{1}, \quad m_{\Lambda}^{2}=-\frac{c_{2}}{c+c_{1}} .
$$

Введем для удобства параметр $\Lambda_{1}^{2}=c \Lambda^{2}$, который будем считать фиксированным. Коэффициенты $c_{n}<0$, поэтому условие отсутствия тахиона есть $\Lambda<\Lambda_{1} / \sqrt{-c_{1}}$. Нефиксированным параметром модели при заданном числе петель является один параметр $\Lambda$. Тогда с учетом (37) имеем

$$
\alpha_{\text {syn }}\left(Q^{2}\right)=\alpha^{\mathrm{pt}}\left(Q^{2}\right)+\frac{4 \pi}{b_{0}}\left[c_{3}-\frac{c_{2}^{2} \Lambda^{2}}{\Lambda_{1}^{2}+c_{1} \Lambda^{2}}\right]\left(\frac{\Lambda^{2}}{Q^{2}}\right)^{3}+O\left(\left(Q^{2}\right)^{-4}\right) .
$$

Как видно из (38), лидируюший степенной непертурбативный член при $Q^{2} \rightarrow \infty$ быстро убывает и при отсутствии тахиона является отрицательным ${ }^{6)}$. Для массового параметра $m_{\mathrm{g}}$ (который мы мы называем динамической массой глюона) из уравнений $(37)$ получаем

$$
m_{\mathrm{g}}=\Lambda \sqrt{\frac{-c_{2} \Lambda^{2}}{\Lambda_{1}^{2}+c_{1} \Lambda^{2}}}
$$

Обратимся к интерпретации параметра $\Lambda_{1}=\sqrt{c} \Lambda$, характеризуюшего величину сингулярного члена. Как уже отмечалось, поведение инвариантного заряда $\alpha_{\mathrm{V}} \sim 1 / Q^{2}$ при

\footnotetext{
5) Это требование соответствует принципу минимальности непертурбативных вкладов в пертурбативной УФ-области [23].

6) Отрицательным оказывается при больших $Q^{2}$ и весь непертурбативный “хвост" бегущей константы связи $\alpha_{\mathrm{syn}}\left(Q^{2}\right)$.
} 
$Q^{2} \rightarrow 0$ в “замороженной” КХД соответствует линейному запираюшему кварк-антикварковому статическому потенциалу, определяемому через вакуумное ожидание петли Вильсона. Соответствие потенциала и инвариантного заряда мы устанавливаем с помощью формулы

$$
V(r)=-\left.\frac{4}{3} \int \frac{d^{n} q}{(2 \pi)^{n}} e^{i \mathbf{q r}} \frac{4 \pi \alpha_{\mathrm{V}}\left(q^{2}\right)}{q^{2}}\right|_{n=3}
$$

- определения $\alpha_{\mathrm{V}}\left(q^{2}\right)$ как эффективного заряда, который является схемно-независимой калибровочно-инвариантной величиной $[1],[6],[26]$. Цветовой фактор соответствует группе $S U\left(N_{c}\right), N_{c}=3$. Пусть в инфракрасной области

$$
\alpha_{\mathrm{V}}\left(q^{2}\right) \simeq \frac{3}{2} \frac{\sigma}{q^{2}}, \quad q^{2} \rightarrow 0
$$

Тогда интеграл по трехмерному импульсному пространству в формуле (40) формально расходится в нуле. Мы доопределяем его, вводя размерную регуляризацию. После интегрирования сингулярного члена по $n$-мерному евклидову импульсному пространству мы переходим к $n=3$. Тогда, поскольку при этом расходимостей не возникает, переход к $n$-мерному интегрированию дает не только регуляризацию, но и доопределение формально расходяшегося интеграла для случая $n=3$. В результате для ИК-поведения эффективного заряда (41) поведение потенциала на больших расстояниях получается следующим:

$$
V(r) \simeq \sigma r, \quad r \rightarrow \infty
$$

где $\sigma \equiv a^{2}$ - параметр натяжения струны. Определим параметр $\Lambda_{1}$ синтетической модели $\alpha_{\text {syn }}$ из условия соответствия сингулярного в нуле члена в формуле (35) ИК-поведению (41) эффективного заряда $\alpha_{\mathrm{V}}$. Тогда

$$
\frac{3}{2} \sigma=\frac{4 \pi}{b_{0}} \Lambda_{1}^{2}, \quad \Lambda_{1}^{2}=c \Lambda^{2}
$$

Задав параметр натяжения струны, из формулы (43) мы можем фиксировать параметр модели $\Lambda_{1}$. Тогда при $a \simeq 0.42$ ГэВ,$b_{0}=9$ получаем ${ }^{7)} \Lambda_{1} \simeq 435 \mathrm{MэВ.} \mathrm{Параметр} \mathrm{мо-}$ дели $\Lambda$ (следовательно, и параметр $c$ ) может быть фиксирован условием нормировки, и тогда модель будет полностью фиксирована.

Рассмотрим зависимость динамической массы глюона от значений параметра $\Lambda$ при различном числе петель исходного пертурбативного приближения. На рис. 1 приведены четыре кривые для соответствующих зависимостей $m_{\mathrm{g}}(\Lambda)$ при $\Lambda_{1}=435 \mathrm{MэВ.} \mathrm{До}$ 400 МэВ кривые не слишком сильно расходятся, и $m_{\mathrm{g}} \simeq 0.6$ ГэВ при $\Lambda=375$ МэВ. При проведении численных расчетов здесь и далее мы полагаем $n_{f}=3$.

Исследуем импульсную зависимость синтетической модели бегушей константы связи $\alpha_{\text {syn }}\left(Q^{2}\right)$ и сравним ее с $\alpha_{\text {an }}\left(Q^{2}\right)$ и $\alpha^{\mathrm{pt}}\left(Q^{2}\right)$. Нормируем эти величины на массе $\tau$-лептона

\footnotetext{
7) Такое значение параметра натяжения струны получается, в частности, в модели релятивистской струны с точечными массивными кварками на концах [31]. Тогда наклон траекторий Редже $\alpha^{\prime}=1 /(2 \pi \sigma) \simeq 0.90 \Gamma_{\ni} \mathrm{B}^{-2}$.
} 


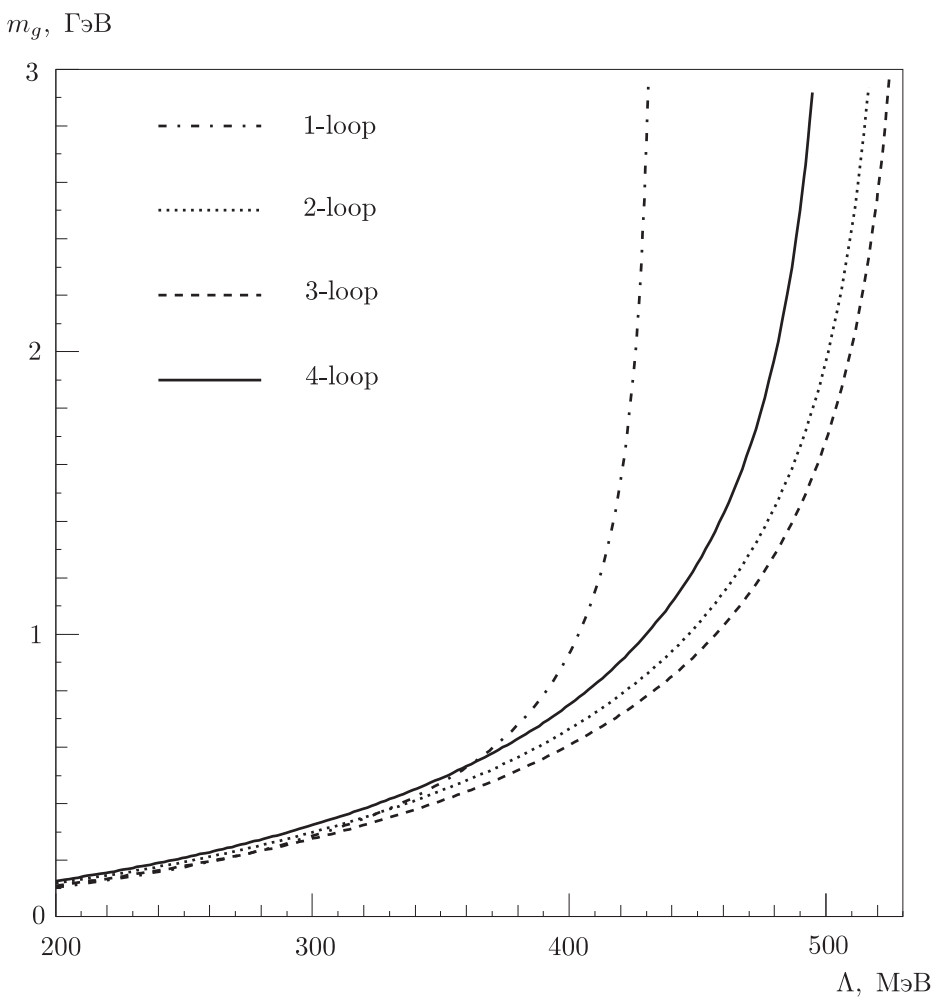

Рис. 1. Динамическая масса глюона $m_{\mathrm{g}}$ как функция параметра $\Lambda$ для различного числа петель исходного приближения теории возмущений.

ТАБлицА 2. Параметры $\Lambda_{\mathrm{pt}}$ (МэB), $\Lambda_{\mathrm{an}}(\mathrm{MэB}), \Lambda_{\mathrm{syn}}(\mathrm{MэB})$, динамическая масса глюона $m_{\mathrm{g}}(\mathrm{MэВ)} \mathrm{и} \mathrm{параметры} c, d$ в зависимости от числа петель. Число ароматов активных кварков $n_{f}=3$, $\Lambda_{1}=435$ МэВ. Условие нормировки: $\alpha_{s}\left(M_{\tau}^{2}\right)=0.323, M_{\tau}=1.777$ ГэВ.

\begin{tabular}{|c|c|c|c|c|}
\hline & 1-loop & 2-loop & 3-loop & 4-loop \\
\hline$\Lambda_{\mathrm{pt}}$ & 204.65 & 381.89 & 351.07 & 344.82 \\
\hline$\Lambda_{\text {an }}$ & 240.46 & 599.22 & 494.71 & 505.31 \\
\hline$\Lambda_{\text {syn }}$ & 204.65 & 383.43 & 351.89 & 345.90 \\
\hline$m_{\mathrm{g}}$ & 109.01 & 580.34 & 416.06 & 473.66 \\
\hline$c$ & 4.5247 & 1.2890 & 1.5304 & 1.5839 \\
\hline$d$ & 3.5247 & 0.6230 & 0.8806 & 0.8558 \\
\hline
\end{tabular}


условием $\alpha_{s}\left(M_{\tau}^{2}\right)=0.323, M_{\tau}=1.777$ ГэВ. Такое значение константы сильного взаимодействия в точке $M_{\tau}$ приводится в работе [14] в качестве среднего значения при различных способах ее определения. Соответствующие этому условию нормировки значения параметров $\Lambda$, динамической массы глюона $m_{\mathrm{g}}$ и параметров $c$ и $d$ приведены в табл. 2. Отметим два обстоятельства. Параметры $\Lambda_{\mathrm{syn}}$ и $\Lambda_{\mathrm{pt}}$ весьма близки друг к

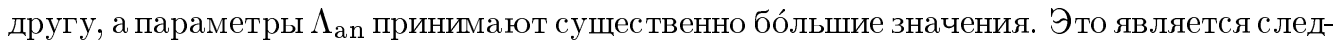
ствием наложения условий (37), обеспечиваюших быстрое убывание непертурбативных членов в $\alpha_{\mathrm{syn}}\left(Q^{2}\right)$. Для всех рассматриваемых величин однопетлевой случай оказывается выделенным, а далее с ростом числа петель наблюдается стабилизация. На рис. 2 для различного числа петель приведены кривые $\alpha_{\mathrm{syn}}, \alpha_{\mathrm{an}}$ и $\alpha^{\mathrm{pt}}$ как функции импульса $Q$ при значениях $Q$ от нуля до 2 ГэВ.

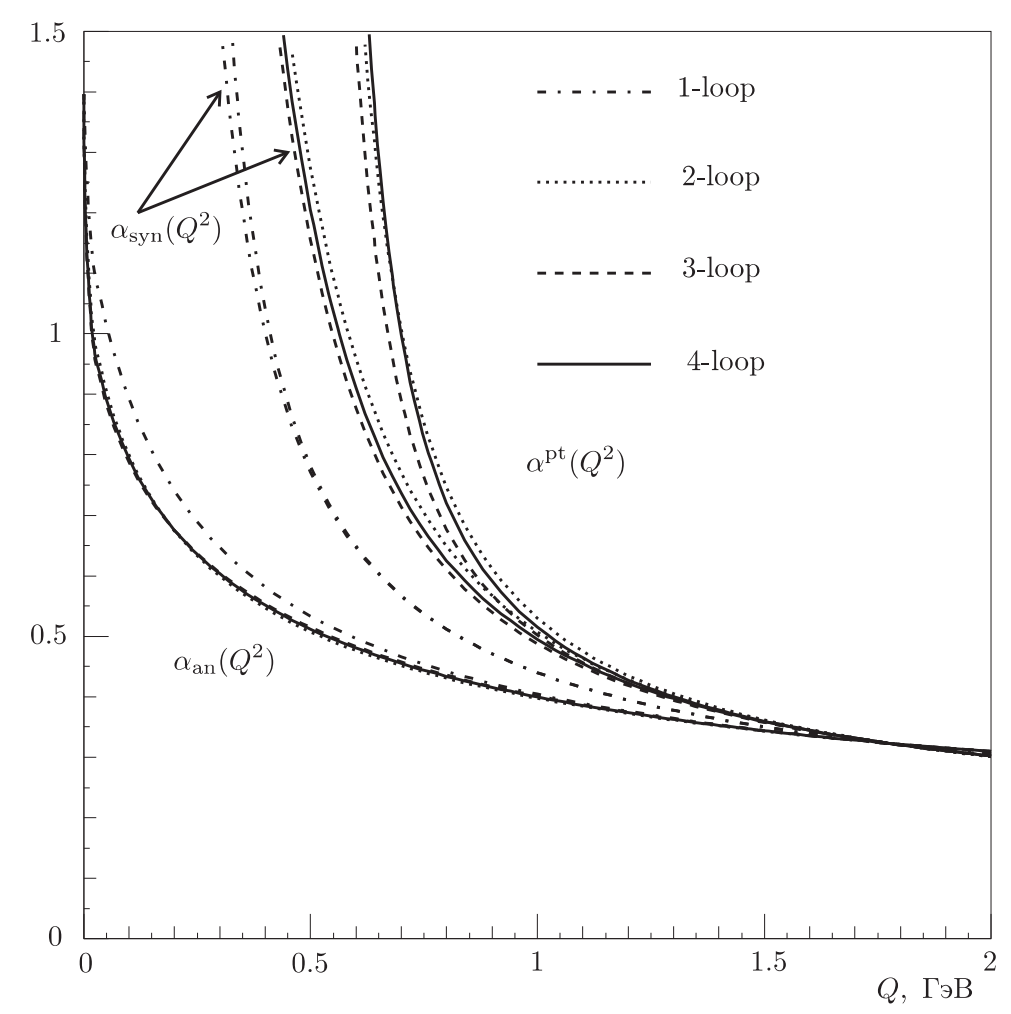

Рис. 2. Величины $\alpha^{\mathrm{pt}}\left(Q^{2}\right), \alpha_{\mathrm{an}}\left(Q^{2}\right)$ и $\alpha_{\mathrm{syn}}\left(Q^{2}\right)$ как функции импульса $Q$ для 1-4-петлевых случаев. Условие нормировки для всех функций $\alpha_{s}\left(M_{\tau}^{2}\right)=0.323, M_{\tau}=1.777$ ГэВ.

Для каждого числа петель пертурбативная кривая проходит выше кривой модели (при $Q<M_{\tau}$ ), которая, в свою очередь, проходит выше аналитической кривой. Посмотрим на петлевую стабильность приведенных кривых. В зависимости от числа петель кривые для модели отличаются значительно меньше, чем пертурбативные кривые. Если исключить однопетлевой случай, то, например, при $Q=0.7$ ГэВ разброс пертурбативных кривых составляет 0.11 , аналитических -0.006 , а кривых модели -0.06 . Таким образом, импульсная зависимость модели обладает высокой петлевой стабильностью. 
ТАБлицА 3. Динамическая масса глюона $m_{\mathrm{g}}$ (МэВ) в зависимости от числа петель и условий нормировки. Число ароматов активных кварков $n_{f}=3, \Lambda_{1}=435 \mathrm{MэB.} \mathrm{Условия} \mathrm{нормировки:}$ $\alpha_{\mathrm{syn}}\left(M_{\tau}^{2}\right)=0.32,0.325, \ldots, 0.36$ при $M_{\tau}=1.777$ ГэВ.

\begin{tabular}{|c|c|c|c|c|}
\hline$\alpha_{\text {syn }}\left(M_{\tau}^{2}\right)$ & 1-loop & 2-loop & 3-loop & 4-loop \\
\hline 0.32 & 104.09 & 553.68 & 397.81 & 452.59 \\
\hline 0.325 & 112.38 & 598.87 & 428.65 & 488.22 \\
\hline 0.33 & 121.14 & 648.09 & 461.66 & 526.57 \\
\hline 0.335 & 130.40 & 702.03 & 497.09 & 567.99 \\
\hline 0.34 & 140.20 & 761.59 & 535.23 & 612.93 \\
\hline 0.345 & 150.56 & 827.96 & 576.45 & 661.94 \\
\hline 0.35 & 161.51 & 902.79 & 621.20 & 715.75 \\
\hline 0.355 & 173.09 & 988.39 & 670.05 & 775.29 \\
\hline 0.36 & 185.36 & 1088.19 & 723.73 & 841.80 \\
\hline
\end{tabular}

ТАБлицА 4 . Непертурбативная компонента $\alpha_{\mathrm{syn}}^{\mathrm{npt}}\left(M_{\tau}^{2}\right)$ в зависимости от числа петель. Число ароматов активных кварков $n_{f}=3, \Lambda_{1}=435 \mathrm{MэВ.}$ Условия нормировки: $\alpha_{\mathrm{syn}}\left(M_{\tau}^{2}\right)=0.32,0.325, \ldots, 0.36$ при $M_{\tau}=1.777$ ГэВ.

\begin{tabular}{|c|c|c|c|c|}
\hline$\alpha_{\text {syn }}\left(M_{\tau}^{2}\right)$ & 1-loop & 2-loop & 3-loop & 4-loop \\
\hline 0.32 & -0.000004 & -0.000718 & -0.000389 & -0.000530 \\
\hline 0.325 & -0.000005 & -0.000879 & -0.000472 & -0.000645 \\
\hline 0.33 & -0.000006 & -0.001074 & -0.000572 & -0.000781 \\
\hline 0.335 & -0.000007 & -0.001308 & -0.000689 & -0.000943 \\
\hline 0.34 & -0.000009 & -0.001590 & -0.000829 & -0.001136 \\
\hline 0.345 & -0.000011 & -0.001932 & -0.000994 & -0.001366 \\
\hline 0.35 & -0.000013 & -0.002347 & -0.001190 & -0.001638 \\
\hline 0.355 & -0.000016 & -0.002853 & -0.001421 & -0.001963 \\
\hline 0.36 & -0.000019 & -0.003474 & -0.001695 & -0.002352 \\
\hline
\end{tabular}

Рассмотрим следуюший интервал допустимых [13]- 115$]$ значений $\alpha_{s}\left(M_{\tau}^{2}\right): \alpha_{s}\left(M_{\tau}^{2}\right)=$ $0.32 \div 0.36$. Для этого интервала значений инвариантного заряда в точке $M_{\tau}$ характер петлевой зависимости всех рассматриваемых параметров соответствует табл. 2. 
В табл. 3 приведены значения динамической массы глюона $m_{\mathrm{g}}$ в зависимости от числа петель при условиях нормировки $\alpha_{\text {syn }}\left(M_{\tau}^{2}\right)=0.32,0.325, \ldots, 0.36$. Видно, что при увеличении значения $\alpha_{\text {syn }}$ в точке нормировки $M_{\tau}$ как сами массы $m_{\mathrm{g}}$, так и их разброс в зависимости от числа петель увеличиваются. Согласно формулам (32), (35) мы имеем уравнение

$$
\alpha_{\text {syn }}\left(Q^{2}\right)=\alpha^{\mathrm{pt}}\left(Q^{2}\right)+\alpha_{\mathrm{syn}}^{\mathrm{npt}}\left(Q^{2}\right),
$$

аналогичное уравнению (20) для однопетлевого случая. Оценим величину непертурбативной компоненты синтетического инвариантного заряда. В табл. 4 приведены значения непертурбативной компоненты синтетического инвариантного заряда в точке $M_{\tau}$ при условиях нормировки таких же, как и в табл. 3. Мы видим, что непертурбативная компонента отрицательна и весьма мала. Она является максимальной (по абсолютной величине) в двухпетлевом случае при $\alpha_{\text {syn }}\left(M_{\tau}^{2}\right)=0.36$ и при этом не превышает одного процента. С увеличением $Q^{2}$ непертурбативная компонента убывает как $\sim 1 /\left(Q^{2}\right)^{3}$, так что синтетический инвариантный заряд при $Q \geqslant 1.8$ ГэВ практически совпадает со своей пертурбативной компонентой.

\section{5. ЗАКЛЮЧЕНИЕ}

При построении аналитического инвариантного заряда (31) в КХД нефизические сингулярности теории возмушений в ИК-области исчезают, а в УФ-области возникают непертурбативные степенны́е поправки, которые в сравнении с основной пертурбативной компонентой инвариантного заряда быстро убывают при больших $Q^{2}$. Однако при рассмотрении непертурбативных величин может оказаться, что убывание непертурбативных вкладов не является достаточно быстрым для согласованного определения этих величин. В синтетической модели инвариантного заряда предлагается добиться максимально возможного подавления непертурбативных вкладов при больших $Q^{2}$ с помощью минимального числа членов полюсного типа. Как оказалось, параметры, характеризуюшие введенные дополнительные непертурбативные члены, допускают прозрачную физическую интерпретацию и имеют разумные значения. Мы рассматриваем аналитический инвариантный заряд в однопетлевом случае и вводим два дополнительных члена полюсного типа: сингулярный в нуле член вида $\sim 1 / Q^{2}$, который может соответствовать линейному конфайнменту кварков, и член вида $\sim 1 /\left(Q^{2}+m_{\mathrm{g}}^{2}\right)$, в котором параметр $m_{\mathrm{g}}$ может иметь смысл ненулевой динамической массы глюона [32]. Потребовав, чтобы убывание непертурбативных членов при больших $Q^{2}$ было максимально возможным, мы приходим к модели вида (1). Инвариантный заряд вида (1), построенный на основе аналитического инвариантного заряда (5), мы называем однопетлевой синтетической моделью инвариантного заряда, поскольку он включает параметры, относяшиеся как к УФ-, так и к ИК-области. Модель $\alpha_{\mathrm{syn}}^{(1)}\left(Q^{2}\right)$ имеет два независимых параметра. Это размерный параметр $\Lambda$ и безразмерный параметр $c$ (или же еще один размерный параметр $\Lambda_{1}=\sqrt{c} \Lambda$ ), характеризуюший величину сингулярного члена. В разделе 2 рассмотрены свойства однопетлевой синтетической модели инвариантного заряда и его непертурбативной компоненты в зависимости от значений безразмерного параметра $c$. Записано дисперсионное соотношение (8), (9), (7) и исследована бета-функция модели (14).

В результате исследования многопетлевого аналитического инвариантного заряда и его непертурбативной компоненты возникает возможность естественного обобщения 
синтетической модели инвариантного заряда на многопетлевые случаи. Многопетлевая синтетическая модель инвариантного заряда (35) строится точно так же, как и однопетлевая - путем введения двух дополнительных непертурбативных членов вида $\sim 1 / Q^{2}$ и $\sim 1 /\left(Q^{2}+m_{\mathrm{g}}^{2}\right)$. Требование минимальности непертурбативных вкладов приводит к двум уравнениям (37) для параметров введенных непертурбативных членов. В результате модель, как и в однопетлевом случае, имеет два независимых параметра. Это параметр $\Lambda$, который в силу весьма быстрого убывания непертурбативных вкладов при больших $Q^{2}$ практически равен параметру $\Lambda_{\mathrm{QCD}}$ в области применимости пертурбативных решений. Второй параметр модели - безразмерный параметр $c$ (или размерный параметр $\Lambda_{1}=\sqrt{c} \Lambda$ ), характеризуюший сингулярный член. Если величину параметра ИК-усиления модели посредством формул (43) сопоставить параметру натяжения струны струнных моделей, то мы приходим к формуле (39) для динамической массы глюона как функции параметра $\Lambda$. Соответствуюшие зависимости для различного числа петель при $\Lambda_{1}=435 \mathrm{MэB}$ (соответственно $\sigma^{1 / 2}=0.42$ ГэВ) приводятся на рис. 1 . Нормировка окончательно фиксирует синтетическую модель инвариантного заряда; значения параметров модели приведены в табл. 2, 3. Соответствующие импульсные зависимости синтетической модели инвариантного заряда в сравнении с пертурбативным инвариантным зарядом и аналитическим инвариантным зарядом приведены на рис. 2. Параметр $m_{\mathrm{g}}$ при $\Lambda<\Lambda_{1} / \sqrt{-c_{1}}$ является действительным, и тогда синтетический инвариантный заряд $\alpha_{\text {syn }}\left(Q^{2}\right)$ является голоморфной функцией в комплексной плоскости $Q^{2}$ с разрезом вдоль отрицательной действительной полуоси. Это выполняется для всех значений $\alpha_{\text {syn }}\left(M_{\tau}^{2}\right)$ из рассмотренного интервала $0.32 \div 0.36$ для $1-4$-петлевых случаев.

Таким образом, синтетический инвариантный заряд КХД $\alpha_{\mathrm{syn}}\left(Q^{2}\right)$ для $1-4$-петлевых приближений исходной теории возмушений имеет следующие свойства.

- Как функция $Q^{2}$ он имеет аналитическую структуру, соответствующую условию причинности, т.е. является голоморфной функцией в комплексной плоскости $Q^{2}$ с разрезом вдоль действительной оси от нуля до $-\infty$.

- Как функция значения в точке нормировки $\alpha_{\text {syn }}\left(\mu^{2}\right)$ он имеет сушественную особенность в нуле, асимптотическое разложение его непертурбативной части по $\alpha_{\text {syn }}\left(\mu^{2}\right)$ при $\alpha_{\text {syn }}\left(\mu^{2}\right) \rightarrow+0$ равно нулю, что дает соответствие исходной теории возмущений.

- В УФ-области он соответствует результатам теории возмущений с точностью до быстроубывающих степенны́х непертурбативных членов вида $\sim 1 /\left(Q^{2}\right)^{3}$ при $Q^{2} \rightarrow \infty$.

- В ИК-области он не имеет нефизических сингулярностей теории возмушений. Имеются член, соответствующий линейному конфайнменту, и массовый член.

Как видно из табл. 2, 3, при рассмотренном способе фиксации двух параметров модели наблюдается высокая петлевая стабильность (однопетлевой случай является особым) и петлевая стабилизация всех остальных параметров модели. В частности, динамическая масса глюона $m_{\mathrm{g}}$ при условии нормировки $\alpha_{\text {syn }}\left(M_{\tau}^{2}\right)=0.33 \div 0.35$ принимает значения $m_{\mathrm{g}} \simeq 500-700$ МэВ. Таким образом, идентификация параметра модели при сингулярном члене с параметром натяжения струны струнных моделей дает согласованные значения для всех параметров модели. Как видно из табл. 4, непертурбативная компонента синтетического инвариантного заряда при $Q^{2}=M_{\tau}^{2}$ составляет менее од- 
ного процента и при увеличении $Q^{2}$ очень быстро вымирает. Поэтому условия сшивания синтетического инвариантного заряда на порогах тяжелых кварков, например, по непрерывности приведут к соответствию $\alpha_{\mathrm{S}}\left(M_{\tau}^{2}\right) \leftrightarrow \alpha_{\mathrm{S}}\left(M_{Z}^{2}\right)$, близкому чисто пертурбативному случаю.

Согласно формуле $(38)$ при $\Lambda \neq \Lambda_{1} / \sqrt{-c_{1}}\left(c \neq-c_{1}\right)$ непертурбативные вклады при больших $Q^{2}$ убывают как $\sim 1 /\left(Q^{2}\right)^{3}$, что является достаточным для обеспечения сходимости глюонного конденсата в УФ-области [24]. В этой связи представляет интерес обобщение исследования глюонного конденсата (см. [24]) на многопетлевые случаи и сравнение с результатами работы [33] для сингулярной в нуле модели инвариантного заряда, в которой по построению полностью отсутствуют непертурбативные вклады при $Q^{2}>q_{0}^{2}$, где параметр $q_{0} \sim 1$ ГэВ соответствует границе пертурбативной и непертурбативной областей.

Благодарности. Автор выражает глубокую признательность Б. А. Арбузову, А. В. Киселеву, В. А. Петрову, В. Е. Рочеву за полезные обсуждения, а также благодарит Д. В. Ширкова за ценные советы и стимулирующие обсуждения. Работа выполнена при частичной поддержке РФФИ (грант № 05-01-00992).

\section{Список литературы}

[1] Н. Н. Боголюбов, Д. В. Ширков. Введение в теорию квантованных полей. М.: Наука, 1984.

[2] P. J. Redmond. Phys. Rev. 1958. V. 112. P. 1404; H. Н. Боголюбов, А. А. Логунов, Д. В. Ширков. ЖЭТФ. 1959. Т. 37. С. 805.

[3] D. V. Shirkov, I. L. Solovtsov. JINR Rapid Comm. 1996. V. 2[76]-96. P. 5; hep-ph/9604363; Phys. Rev. Lett. 1997. V. 79. P. 1209.

[4] Yu. L. Dokshitzer, G. Marchesini, B. R. Webber. Nucl. Phys. B. 1996. V. 469. P. 93.

[5] И. Л. Соловиов, Д. В. Ширков. ТМФ. 1999. Т. 120. С. 482; hep-ph/9909305.

[6] Д. В. Ширков. ТМФ. 2003. Т. 136. С. 3; hep-th/0210013.

[7] B. A. Magradze. Int. J. Mod. Phys. A. 2000. V. 15. P. 2715; QCD coupling up to third order in standard and analytic perturbation theories. Commun. JINR. E2-2000-222. Dubna, 2000; hep-ph/0010070; Д. С. Курашев, Б. А. Маградзе. ТМФ. 2003. Т. 135. С. 95; hep-th/0104142.

[8] A. I. Alekseev. Phys. Rev. D. 2000. V. 61. P. 114005; hep-ph/9906304.

[9] A. I. Alekseev. On dependence of nonperturbative contributions in $\bar{\alpha}_{s}\left(q^{2}\right)$ on an initial approximation of perturbation theory in an analytic approach to QCD. In: Proceedings of the XVth Workshop on High Energy Physics and Quantum Field Theory. Tver, Russia, 2000. Eds. М. N. Dubinin, V. I. Savrin. М.: НИИЯ

[10] А. И. Алексеев. ЯФ. 2002. Т. 65. С. 1722.

[11] A. I. Alekseev. J. Phys. G. 2001. V. 27. P. L117; hep-ph/0105338.

[12] A. I. Alekseev. Few-Body Syst. 2003. V. 32. P. 193; hep-ph/0211339.

[13] S. Eidelman, K. G. Hayes, K. A. Olive et al. Phys. Lett. B. 2004. V. 592. P. 1.

[14] S. Bethke. J. Phys. G. 2000. V. 26. P. R27; hep-ex/0004021.

[15] O. Biebel. Phys. Rep. 2001. V. 340. P. 165; A. A. Pivovarov. Determination of the numerical value for the strong coupling constant from tau decays in perturbative QCD. Препринт INR-TH-03-3. М.: ИЯИ, 2003; hep-ph/0301074; Z. Phys. С. 1992. V. 53. Р. 461; Nuovo Cimento. A. 1992. V. 105. P. 813.

[16] Ю. А. Симонов. Письма в ЖКЭТФ. 1993. Т. 57. С. 525; ЯФ. 1995. Т. 58. С. 107; 2002. T. 65 . C. $135 ; 2003$. T. 66 . C. 794.

[17] Д. В. Ширков. ТМФ. 2002. Т. 132. С. 484.

[18] A. V. Nesterenko. Mod. Phys. Lett. A. 2000. V. 15. P. 2401; Phys. Rev. D. 2000. V. 62. P. 094028; Int. J. Mod. Phys. A. 2003. V. 18. P. 5475; A. V. Nesterenko, I. L. Solovtsov. 
Mod. Phys. Lett. A. 2001. V. 16. P. 2517; V. Gogohia. Phys. Lett. B. 2004. V. 584. P. 225; hep-ph/0304163.

[19] G. Burgio, F. Di Renzo, G. Marchesini, E. Onofri. Phys. Lett. B. 1998. V. 422. P. 219 ; Nucl. Phys. Proc. Suppl. 1998. V. 63. P. 805; G. Burgio, F. Di Renzo, C. Parrinello, C. Pittori. Nucl. Phys. Proc. Suppl. 1999. V. 73. P. 623; V. 74. P. 388; Ph. Boucaud, G. Burgio, F. Di Renzo et al. JHEP. 2000. V. 0004. P. 006.

[20] L. von Smekal, A. Hauck, R. Alkofer. Ann. Phys. 1998. V. 267. P. 1; Erratum. V. 269. P. 182 ; hep-ph $/ 9707327$; C. D. Roberts, S. M. Schmidt. Prog. Part. Nucl. Phys. 2000. V. 45. P. S1; nucl-th/0005064; R. Alkofer, L. von Smekal. Phys. Rep. 2001. V. 353. P. 281; hep-ph/0007355.

[21] А. И. Алексеев. ЯФ. 1981. Т. 33. С. 516; А. И. Алексеев, Б. А. Арбузов, В. А. Байков. ТМФ. 1982. Т. 52. С. 187; А. И. Алексеев, В. Ф. Еднерал. ЯФ. 1987. Т. 45. С. 1105

[22] A. I. Alekseev. QCD running coupling: freezing versus enhancement in the infrared region. В сб.: Proc. of the XII International Workshop on High Energy Physics and Quantum Field Theory. Samara, Russia, 1997. Ред. В. В. Levtchenko. М.: НИИЯФ МГУ, 1999. С. 334; Препринт IНЕР 97-90. Протвино: ИФВЭ, 1997; hep-ph/9802372.

[23] А. И. Алексеев, Б. А. Арбузов. ЯФ. 1998. Т. 61. С. 314; A. I. Alekseev, B. A. Arbuzov. Mod. Phys. Lett. A. 1998. V. 13. P. 1447.

[24] A. I. Alekseev. The model for QCD running coupling constant with dynamically generated mass and enhancement in the infrared region. In: Proc. of the Workshop on Nonperturbative Methods in Quantum Field Theory, Adelaide, Australia, 1998. Eds. A. V. Schreiber, A. G. Williams, A. W. Thomas. Singapore: World Scientific, 1998. P. 104; Препринт IHEP 98-41. Протвино: ИФВЭ, 1998; hep-ph/9808206.

[25] E. Eichten, K. Gottfried, T. Kinoshita, K. D. Lane, T.-M. Yan. Phys. Rev. D. 1978. V. 17. P. 3090; 1980. V. 21. P. 203; J. L. Richardson. Phys. Lett. B. 1979. V. 82. P. 272.

[26] W. Buchmüller, G. Grunberg, S.-H. H. Tye. Phys. Rev. Lett. 1980. V. 45. P. 103; W. Buchmüller, S.-H. H. Tye. Phys. Rev. D. 1981. V. 24. P. 132.

[27] V. V. Kiselev, A. E. Kovalsky, A. I. Onishchenko. Phys. Rev. D. 2001. V. 64. P. 054009; B. В. Киселев, А. К. Лиходед. УФН. 2002. Т. 172. С. 497; V. V. Kiselev, A. K. Likhoded, O. N. Pakhomova, V. A. Saleev. Phys. Rev. D. 2002. V. 65. P. 034013; V. 66. P. 034030.

[28] D. J. Gross, F. Wilczek. Phys. Rev. Lett. 1973. V. 30. P. 1343; H. D. Politzer. Phys. Rev. Lett. 1973. V. 30. P. 1346

[29] D. R. T. Jones. Nucl. Phys. B. 1974. V. 75. P. 531; W. E. Caswell. Phys. Rev. Lett. 1974. V. 33. P. 244; Э. III. Егорян, О. В. Тарасов. ТМФ. 1979. T. 41. C. 26; O. V. Tarasov, A. A. Vladimirov, A. Yu. Zharkov. Phys. Lett. B. 1980. V. 93. P. 429; S. A. Larin, J. A. M. Vermaseren. Phys. Lett. B. 1993. V. 303. P. 334; T. van Ritbergen, J. A. M. Vermaseren, S. A. Larin. Phys. Lett. B. 1997. V. 400. P. 379.

[30] K. G. Chetyrkin, B. A. Kniehl, M. Steinhauser. Rhys. Rev. Lett. 1997. V. 79. P. 2184.

[31] L. D. Soloviev. Phys. Rev. D. 1998. V. 58. P. 035005; 2000. V. 61. P. 015009.

[32] J. M. Cornwall. Phys. Rev. D. 1982. V. 26. P. 1453.

[33] A. I. Alekseev, B. A. Arbuzov. Mod. Phys. Lett. A. 2005. V. 20. P. 103; hep-ph/0411339.

Поступила в редакцию 21.III.2005 г., после доработки 27.IV.2005 г. 